\title{
Thematic Analysis of the Raters' Experiences Administering Scales to Assess Depression and Suicide in Arab Schizophrenia Patients
}

\section{Iman Amro}

Weill Cornell Medicine - Qatar

\section{Suhaila Ghuloum}

Hamad Medical Corporation

\section{Samer Hammoudeh}

Weill Cornell Medicine - Qatar

Yahya Hani

Hamad Medical Corporation

Arij Yehya

Weill Cornell Medicine - Qatar

Hassen Al-Amin ( $\nabla$ haa2019@qatar-med.cornell.edu )

Weill Cornell Medicine in Qatar https://orcid.org/0000-0001-6358-1541

Research article

Keywords: cultural adaptation, thematic analysis, suicide, depression, schizophrenia, Arabs

Posted Date: March 10th, 2021

DOI: https://doi.org/10.21203/rs.3.rs-296461/v1

License: (9) (i) This work is licensed under a Creative Commons Attribution 4.0 International License. Read Full License 


\section{Abstract}

Background: This study aimed to enhance the cultural adaptation and training on administering the Arabic versions of the Calgary Depression Scale in Schizophrenia (CDSS) and The International Scale for Suicidal Thinking (ISST) to Arab schizophrenia patients in Doha, Qatar.

Methods: We applied the qualitative thematic analysis of the focus group discussions with clinical research coordinators (CRCs). Five CRCs met with the principal investigator for two sessions; we transcribed the conversations and analyzed the content.

Results: This study revealed one set of themes related to the scales themselves, like the role of the clinician-patient relationship during administration, the semantic variations in Arabic dialects, and the design of scales to assess suicide and differentiate between negative symptoms and depression. The other set of themes is relevant to the sociocultural domains in Muslim Arabs, covering religion, families' role, and stigma. It also covered the approaches to culturally sensitive issues like suicide, taboos in Islam, and the gender roles in Arab countries and its impact on the patients' report of their symptoms.

Conclusions: Our results highlight several cultural and religious aspects to tackle when approaching schizophrenia patients through in-depth discussions and training to improve the assessment tools and treatment services' validity.

\section{Background}

Schizophrenia is a chronic mental disorder with a lifetime prevalence of $0.7 \%$ of the population worldwide [1]. People with schizophrenia are at increased risk of having depression and suicide [2]. Depression is a common comorbidity in patients with schizophrenia and a well-known factor that affects their quality of life [3-5]. Depressive symptoms are associated with cognitive impairments, deterioration of psychosocial functions, extended hospitalization, and increased risk of suicide [6]. The reported rate of depression among schizophrenia patients is $25 \%$ [7-9]. The Calgary Depression Scale in Schizophrenia (CDSS) is a valid instrument for assessing depressive symptoms in schizophrenia [10], independently of the negative or extrapyramidal symptoms [11]. CDSS has been adapted and validated in several countries and cultures [12-16]. We already established the translation and cultural adaptation of the Arabic version of CDSS in Qatar's Arab population [17].

Schizophrenia patients are also at increased risk of attempting suicide [18, 19], and an estimated 10\% commit suicide during their lifetime $[3,20]$. Universally, suicide is a behavior linked to different biopsychosocial factors [21] depending on ethnicity and culture. For instance, the sociodemographic and cultural factors for suicide in Asia were different from the Western countries; however, there were no changes in the clinical characteristics [22]. The International Suicide Prevention Trial (InterSePT) Scale for Suicidal Thinking (ISST) consists of 12 items to assess the current suicidal ideation in patients with schizophrenia [23]. This tool was designed to study clozapine and olanzapine effects on suicidal behaviors in patients with schizophrenia or schizoaffective disorder [24]. The Arabic version of ISST has 
also been validated to assess the severity of suicidal ideation in Arab schizophrenia patients residing in Qatar [25].

The proper identification of the psychiatric signs and symptoms associated with schizophrenia is challenging [26], especially when using universal psychometric measurements and tools originated in a different cultural context. Researchers agree that psychiatric assessment depends on the clinical components and the subjective expressions related to the emotional states and the interpretations of the patients' personal experiences and issues [27]. Subjective experiences are quite challenging to elicit from patients with mental health conditions, who might have language and memory problems. However, there is evidence that people with mental health conditions can reliably and validly report their experiences [28, 29]. Culture can influence the various aspects of psychiatric illness like the presentation, behavior, assessment, severity of symptoms, and management [30,31]. A culture is a group of behavioral norms and values utilized by a particular community to construct their specific and unique perceptions of the world. Culture encompasses variables like language, traditions, religion, and many more [32]. In Arab countries, Arabs share cultural factors like religion, language, and peculiar beliefs and values, which might affect their subjective experiences with mental illness [33]. Culturally, how people perceive and deal with depressed patients differ according to their beliefs and cultural perspectives. Arab cultural beliefs and practices can be decisive in shaping Arabs perceptions and management of psychiatric disorders [34].

During the quantitative validation of the Arabic CDSS [17] and ISST [25], we underwent focus group discussions with the clinical research coordinators (CRCs) administering these scales. Focus group discussions are a useful and flexible qualitative method that provides information related to participants' experiences $[35,36]$. This paper presents the qualitative analysis of these discussions on the CRCs' experience and the issues they encountered while administering the Arabic versions of ISST and CDSS for Arab schizophrenia patients. These focus group sessions and the analysis aimed to identify and tackle the relevant themes to improve the utility and validity of the Arabic CDSS and ISST in Arab populations.

\section{Methods}

This study was part of a project to translate and culturally adapt scales used to assess patients with schizophrenia. We did the project in Qatar between 2013 and 2015, supported by Qatar National Research Fund (QNRF) (Grant number: NPRP 4-268-3-086).

It was approved by the Institutional Review Boards of both Hamad Medical Cooperation (HMC) and Weill Cornell Medicine (WMC-Q) (Protocol number: 11129/11) in Doha, Qatar.

These focus group sessions and qualitative study were part of the raters' preparation and training administering the scales.

All the subjects involved signed a written informed consent after explaining the study procedures' details and duration. 
The scales were translated and validated to assess Arabic patients with schizophrenia. The information on the translation process, pilot study, and further details on the validation project methods are available in the published papers $[17,25]$.

\section{Study Setting}

Qatar is one of the Arabic Gulf countries in the Middle East, with an estimated population of 2.5 million, of which $28 \%$ are Arabs, including Qatari and non-Qatari [37]. Qatar is a rapidly developing country where most of the population are expatriates, but the Qataris and other Arabs are the most stable population. We conducted the study at the Mental Health Hospital, Department of Psychiatry, HMC, the main psychiatric facility in Qatar. The hospital has four inpatient wards (70 beds with $95 \%$ occupancy) and ten outpatient clinics with about 120 visits daily.

\section{Focus Group Participants And Approach}

We had two focus group sessions (90 minutes each) to discuss the Arabic versions of CDSS and ISST. We held discussions in a comfortable and familiar setting; We used both English and Arabic languages throughout the sessions. Members who participated in the focus group discussions consisted of the CRC's team and the study moderator who facilitated the discussions. All participants were bilingual, speaking English and Arabic. All CRCs were health professionals holding different medical backgrounds, including medical doctors, psychiatrists, clinical psychologists, and nursing, with varied experiences in mental health research, ranging from 3-5 years.

They were first supervised on interviewing subjects and administering scales; each CRC was trained with 20 participants before the actual recruitment of subjects.

The study moderator used a semi-structured topic guided approach (see guide as a supplementary file) to discuss and explore the CRC's feedback about the scales' administration and address the barriers and issues they encountered in the recruitment process. We discussed all comments and feedback about patients' responses as well.

We recorded and transcribed the discussions and took notes related to specific questions and issues during the debate. The moderator adopted the semi-structured approach. He covered all aspects, including the recruitment process, CRCs' experience and encounters, and the scales themselves, by asking about individual items on each scale and the cultural and translational issues.

\section{Data analysis}

Focus group discussions were recorded and subsequently transcribed manually using the English language. We used the thematic analysis approach [38] for this study: 1) We read the transcript notes several times to become familiar with the content and identify primary patterns. The notes were reviewed 
independently by the moderator and an independent reviewer for quality control and to ensure that they are inclusive and comprehensive. 2) using these notes, we coded each item in the data repeatedly; and during the coding process, we manually generated as many codes as possible. 3 ) the initial codes and items were reviewed to identify the themes and subthemes. 4) all themes and subthemes were examined to check the relations and patterns between them. 5) Also, to ensure consistency and coherency, all topics were tested against each other and the original data. Accordingly, some themes were broken into smaller components, while others emerged together as the main themes. During the analysis process, continuous monitoring was done to provide a clear, convincing, and well-organized representation of the data and the topic. 6) Later, all quotes from the group interactions were listed for each theme in a narrative way where appropriate. We did revisions for the final items to ensure that we identified the issues related to the available data. Other team members who were not involved in the translation or focus group discussions were also involved in the analysis to reduce bias and to confirm that we thoroughly analyzed the data, and the themes were consistent and reflective of the discussions. To ensure participants' anonymity, we treated the data with confidentiality by changing all identification data into unique identifiers during the analysis process. However, quotes in the results section appear without any identifiers.

\section{Results}

We will present the themes from the thematic analysis in two sections: 1) those related to the translation, cultural adaptation, and administration of Arabic CDSS and ISST, and 2) themes that are associated with the Arab and Qatari cultures.

\section{Items Related To Scales' Design And Administration}

Design of ISST: One central point related to the ISST design is the lack of instructions on how to deliver the questions. The CRC commented on this point: "when assessing the risk of suicide, the first question asks if the patient has any suicidal thoughts. If the patient answered, NO, I have no suicidal thoughts, you still have to ask the following questions, which are all designed to elaborate more on suicidal thoughts." This series of questions will increase the risk of provoking anxiety in the patient. Also, there are no prompt or explicit instructions to tell the CRCs when to skip the following question or to continue when appropriate. So, the team thought that having clear guidance in place would save time and avoid asking unnecessary items that could impact the patient's cooperation in completing the questionnaires.

CRCs discussed the time frame of the patients' suicidal thoughts. The team questioned the seven-day criteria linked to this scale; they raised the scenario in which a patient had suicidal thoughts $10-15$ days ago but not lately, for instance, wondering if such patients would be automatically excluded from the study. However, this is not the case in the CDSS as it has clear instructions to prepare the CRC and the patient for the next questions. "As per the instructions in CDSS, we do not have to read all the potential answers in advance. We ask, wait, and then we elaborate to get the right answer; hence, it is the answer based on the assessment more than giving scores." Another CRC commented on the differences in time 
frames in the two scales when assessing suicide: "using two different scales to assess the same element with two different time frames will affect the reliability of the patients' responses, which might affect the rating scores. So, we should clarify this to the patient...".

\section{CDSS, self-rating vs. rater's assessment}

The group discussed the differences between the self-rating scales vs. those based on raters' assessment. They agreed that "It is a lot easier to get the answers from the patients by reading all the potential answers among all the scales, and the patients will pick what suit them." Also, they elaborated that with more explanation and re-wording, the final rating will depend on the assessment of each rater, which might introduce more discrepancies among the CRCs' scores.

The group agreed that CDSS is designed to be used by trained raters and not intended for selfassessment. Thus, the best approach is to follow the standard procedure to administer the CDSS, where they read the answers, and then patients can pick their choices without their interference to avoid affecting the final score. However, one CRC commented that: "because it is based on your assessment, again you might have to make explanations, and depending on the answers you decide where they fit; the answer is not yes or no... You have to use your assessment, especially in the last item, as it completely depends on your assessment, not on what the patient says."

CDSS, depression vs. negative symptoms: The group elaborated on how and what in the CDSS to differentiate depressive symptoms from negative symptoms of schizophrenia. Examples of questions used to facilitate the discussion included: "Are you able to differentiate, by using CDSS, between depression and negative symptoms? Do the questions help to differentiate these two entities, or they still look the same for you?" After significant deliberation and guidance from the PI, the group agreed on the following points:

1. "It is very challenging to differentiate between negative symptoms and depression."

2. "The concepts themselves overlap a lot; in depression, the patient could be agitated or could be passive; he could be sleeping a lot, or sleepless; he could be anxious or blocked. The challenge is that depressed patients might feel hopeless, always bored; everything they are doing is wrong, wish to die. But in schizophrenia patients with negative symptoms, they don't tell such things; they will say nothing; if you ask him, he will say, I'm okay, everything is good, and you barely get yes or no answers from them. Even they both might have a flat affect, but the core is different."

3. "Schizophrenia patients suffering from negative symptoms would not talk about depression even they both look the same. You cannot get him to elaborate..."

4. "It is challenging, patients with schizophrenia might get depression separate from the schizophrenia, and this will predispose them to suicide..."

5. "Depressed person is the person who changed from a productive person to someone who is not, while someone experiencing negative symptoms (like alogia, no motivation, no initiative) will be different... will always be like that, and most do not recover..." 


\section{Sensitive Issues In Arab Culture}

One CRC said, "The scales are culturally sensitive...", and he suggested, "spending more time at the beginning of the interview to explain it and to know how you will enter the grey area, this will probably help the patient to feel more comfortable to answer the questions... meaning to prepare the patient for the upcoming questions... and I'm talking about this and that...etc., so that could probably prepare them to the culturally-sensitive matters...".

\section{Rapport with patients}

CRCs discussed the patient-clinician relationship and how that could be essential in developing a connection with the patient. All agreed that in the Arab culture, doctors have the authority to ask, discuss, and get the answers from the patients in a much comfortable way than the CRCs do. "In the clinical setting, if you are a doctor, you have the authority; for example, the patient thinks, "I should tell the truth to the doctor and be honest and say what's on my mind..." They did not feel that this applies to their relationship with patients. However, CRC's pointed out that training and expertise have helped them to quickly develop a rapport with the patients, considering the amount of time they have. So, the question was raised by one of the CRC's "...Can we establish rapport within five minutes with the patient? ..." The group agreed that "... the idea is not to jump and start into the topic if you feel that the patient is not comfortable and relaxed, but yes, he will not trust you and tell you all about his life; also, you need to know that even in the clinical setting, you can't afford more than this time to do so..."

Suicide and religion: ISST was designed to assess suicide among schizophrenia patients. However, suicide is a taboo within the Muslim community. As the subjects were Arabs and Muslims, the CRCs repeated: "It is very challenging to open and discuss this issue with the participants." In response to the question: "Have you ever felt that talking about suicidality is something culturally sensitive?" One CRC replied, "...yes, of course, and the patients are mostly upset... and try to convince us it's not good, and we are not allowed to talk about it. They were always saying phrases related to religion that it's something completely forbidden... reacting defensively. "Another participant added, "1 can't recall someone who was super comfortable talking about this; even in the control group, they flip when you ask them about suicidality, it's always a closed subject for them."

Another interesting point to mention "...even if they experienced this at some point in their life, and although they were admitted with suicidal thoughts, if you ask them, they will say no, so, at this point, you can't ask and tell them no it is okay let's talk about it." Accordingly, the moderator asked, "Did you feel that the patients are hiding the reality, or they were genuine in their answers?" The answer was, "Well, there are some of them who hide, and others are genuine, but down the line, you ask them several questions about the suicidality, and eventually, the reality will come out. I do not think they are comfortable talking about this..."

Also, question 8 in ISST covering the deterrents against attempting suicide might complicate the assessment further as "... we still have to explain it by mentioning religion or family as possible 
deterrents." However, to minimize the cultural sensitivity, the group discussed the approaches to assess suicide in Arab culture without making the patients feel guilty and focusing on their suffering and how the disease can alter the way we think and act on things. The group agreed that building rapport and addressing this matter using a neutral approach might help in the rater's assessment of suicide risk in this population.

CRCs pointed out that Arab and Muslim patients who have a mental illness mostly seek religious healers for blessing and spiritual support. The mental health professional is their last stop to seek professional advice. They confirmed that building rapport and spending more time with subjects was productive, especially when introducing the issue of suicide in a non-threatening and non-judgmental way.

\section{Language- dialects and cultural influence}

CRCs discussed the difficulties they encountered during the scales' administration in terms of language. The spoken Arabic language has many dialects depending on the country of origin. It is common to find one word with several meanings, but "...more so in the various Arabic dialects..." The formal Arabic language is not the same as the spoken dialect in each Arab country. Words can have one meaning in a dialect and have a different sense in another, which makes choosing one form of Arabic translation virtually impossible. Therefore, using the classical "formal" state of the Arabic language was the only way forward to overcome this issue; please refer to (Hammoudeh et al., 2016) [25] for more details about the translation process used for the scales. The classical form of the Arabic language is widely shared, understood, and used on all legal documentation in the Arab world; it was, therefore, easy for our CRCs to modify the written translated scales into acceptable and straightforward forms when interviewing the patients.

The translation of some terms while interviewing the patients was a challenge for the CRCs and the patients. For example, the translation for item 4 in the ISST "passive suicidal ideation" in the Arabic language was not very clear, which "we always have to explain it more and give more detailed examples." Also, in item 7 about "Delusions," patients would understand this word differently; more explanation was always required to provide the right meaning to make it clear for the patient because, in Arabic dialects, there is no one translation for this word. An explanation of having delusions was needed; this is where the importance of receiving professional training was highly emphasized and recommended.

\section{Mental illness and stigma}

The team commented on the stigma in the Arab world, particularly in the Middle East countries where mental health illness is profoundly affected by the culture and the local norms. For example, "...family interference plays a significant role in this region where most of the mental illness cases are not properly managed, simply to avoid being labeled as a psychiatric patient." We extensively discussed stigma as it has affected the recruitment process from the CRC's point of view. One CRC said, "... I have come across this situation more than once, where the patient refused to come for a follow-up interview in the validation project because they want to avoid being near the psychiatric hospital and avoid being spotted 
by someone they know. They always request to meet at the general hospital..." The female CRCs added that this social stigma is more so for women with mental illness where "this stigma is aggravated by the well-known gender disparity in the Arab culture."

\section{Discussion}

This qualitative study utilized the thematic analysis approach to identify the relevant items encountered by the raters administering the Arabic versions of ISST and CDSS during the cultural adaptation of these scales to the Arab population residing in Qatar. Briefly, there are themes related to the scales' original design, the differences between scales rated by patients vs. those based on raters' assessment, and the differentiation between negative symptoms vs. depression by CDSS. The other themes are mostly related to the Arab and Islamic cultures: the role of religion and its impact on the assessment of suicide, the various Arabic dialects used by Arabs and its influence on the ratings, patient-clinician relationships in the Arab culture, and the intense stigma around mental illness among the Arabs especially for women. The translation, cultural adaptation, and validation of the Arabic ISST and CDSS have demonstrated that both scales are valid and reliable among Arab schizophrenia patients [17, 25]. To our knowledge, there are no qualitative studies on these scales in the Arab countries or other nations. Thus, we cannot make direct comparisons between our results and other similar studies. However, we will attempt in the next sections to discuss the social and cultural significance of these themes in the Arab or Islamic cultures and whether such themes or others have been reported when assessing suicide and depression in schizophrenia in other international studies.

We mostly addressed the significant issues related to scales' design through the validation project's training phase. The CRCs received professional training and regular supervision while administering the scales. We also held weekly meetings to discuss their concerns and unify the approach and skills during the recruitment stage, including handling the lack of instructions and questions, the barriers to rapport, and the disclosure regarding suicidality and depression. Concerning the CDSS efficiency to differentiate between negative symptoms and depression among schizophrenia patients, there was a general agreement that experience and professional training on the scales helped the CRCs master the skills and increased their knowledge about these two entities. It is vital for all staff working with schizophrenia patients to receive dedicated training in suicide risk assessment and management. Such training should emphasize the importance of addressing conditions that could be related to or overlapping with depression. This unified approach is crucial to ensure that the entire clinical team is aware of the associated risk factors and how to minimize them $[18,20]$. Therefore, the best approach to address the issues related to the design and administration of these scales is to have extensive training and supervision early on concerning relevance of rapport with subjects, clearing ambiguities in the structure of the scale, and proper differentiation of depression vs. negative symptoms in the Arab patients with schizophrenia.

Previous studies have highlighted the importance of cultural aspects when assessing symptoms of schizophrenia [39]. In this regard, studies from the western countries showed the preponderance of 
depressive symptoms, delusions, and thought broadcasting and insertion, while those from non-Western countries showed more auditory and visual hallucinations [40,41]. Thus, using proper methods for cultural adaptation and translation of scales and qualitative research are essential to adapt scales that consider the social context and self-perception of symptoms [42]. In our study, the CRCs and patients emphasized that religion might play a role in minimizing the disclosure about suicide among schizophrenia patients because the Islamic religious beliefs are deeply rooted in their life, acts, and thoughts; these Islamic values forbid suicide [43]. The Arabic and Islamic values favor the family and religious ties over the individual needs or preferences. Thus, the act of suicide can affect the whole family or tribe $[44,45]$. In general, within the Arabic cultural context, when the mental health symptoms become severe and noticeable by others, families try different kinds of help, usually through religious figures, local healers, and family members. Several months later, they seek help from mental health professionals [34, 46]. However, others suggested that family relations within the Muslim and Arab communities are powerful, impacting the treatment journey for the mental health patient positively [47]. When family relations are stable and supportive, patients who suffer from mental health problems often respond better to their treatment plans. The availability of families can enhance their emotional and economic support and practical support, for example, providing transport to attend appointments, which can improve the patients' coping with their mental illness. These family-patient relationships are also relevant to the assessment and care of schizophrenia in many other cultures $[48,49]$.

Furthermore, studies have shown that religious values are protective factors among Muslims [50, 51]. A study comparing patients with schizophrenia from Hong Kong and Beijing also reported differences in the factors associated with suicide in each population. They concluded that the determinants of suicide attempts in these patients are significantly modulated by the sociocultural factors [52]. Another study reached the same conclusion in another study comparing patients from the United States of America and India [53]. Another aspect of cultural adaptation is the language; the Arabic language is characterized by a vast number of varieties, in which the written standard differs substantially from the spoken vernacular [54]. Arabic speakers often manipulate how they speak based on the context and setting, which can easily impact how patients express their clinical symptoms [55]. In general, CRCs reported that patients with schizophrenia could express their understanding of most questions on both scales. However, particular items needed more clarifications and explanations to get the right answer. Some questions were addressing sensitive issues for Arab patients, like religion and suicide-related problems. Although the themes reported in this study appeared well known to the healthcare professional because of their knowledge and clinical experience, the published literature lacks documentation about the patients' responses and perceptions about the assessment scales and the clinicians' experiences. Other researchers also saw this aspect in other countries; for example, a study showed that the over-diagnosis of schizophrenia in African-Americans was mostly related to cultural and racial differences in assessing psychotic symptoms [56]. The clinician must understand patients' identity and cultural background very well, as culture plays many different roles in the diagnostic process for any illness [57]. To overcome some of the semantic difficulties, the training and professional translation should adhere to the scales' core meaning to minimize the impact of the different dialects and variations in the Arabic language.

Page $10 / 16$ 


\section{Limitations}

This qualitative study had some limitations that are worth discussing. First, the various Arabic countries were not well represented by our sample of subjects and by the CRCs involved, and thus the findings might not be generalized to all Arabs. Therefore, individual studies from each Arab country and with local CRCs from the same country would reflect better the sub-cultures in each Arab country and enhance the clinical measurements in that population. Second, We did no individual interviews with patients or caregivers, and such qualitative data are essential to have the direct input of patients and their families. Such analyses would further understand the relevant cultural specifics when assessing and treating Arab patients with schizophrenia.

\section{Conclusions}

Our findings in this qualitative study covered the CRCs' experiences and the different cultural aspects of Qatar's Arab population. The thematic analysis identified items related to the proper cultural adaptation of psychiatric scales and the importance of training in administering these scales. We should consider the sociocultural context during the early stages of training to help the raters and enhance the validity of the adapted instruments. The qualitative analysis also showed the importance of religion, cultural values, stigma, and social structure when assessing the psychotic symptoms and suicide in Arab patients with schizophrenia. These matters should also be discussed early with the team and unify the approach to handle them properly and improve the assessments with ISST and CDSS. Such qualitative approaches with the raters, patients, and caregivers would guide psychiatry facilities and policymakers to serve schizophrenia patients better.

\section{Abbreviations}

CDSS: Calgary Depression Scale in Schizophrenia; CRC: Clinical research coordinator; HMC: Hamad Medical Cooperation; QNRF: Qatar National Research Fund; ISST: The International Suicide Prevention Trial (InterSePT) Scale for Suicidal Thinking; WCM-Q: Weill Cornell Medicine in Qatar.

\section{Declarations}

\section{Ethics approval and consent to participate}

The study was approved by the Institutional Review Boards of both Hamad Medical Cooperation (HMC) and Weill Cornell Medicine (WMC-Q) (Protocol number: 11129/11) in Doha, Qatar. These focus group sessions and qualitative study were part of the raters' preparation and training administering the scales. All the subjects involved signed a written informed consent after explaining to them the study procedures' details and duration.

\section{Consent for publication}

Not applicable 
Availability of data and materials

To protect the research participants' privacy and confidentiality, our de-identified data are available only upon request and after compliance with the policies and procedures of WCM-Qatar, HMC, and QNRF for data sharing. Requests can be submitted to researchcompliance@qatar-med.cornell.edu.

\section{Competing interests}

All authors declare no commercial or financial conflicts of interest concerning the submitted article.

\section{Funding}

The Qatar National Research Fund (QNRF) thankfully supported this study (NPRP 4-268-3-086). The QNRF did not have any additional role in the study design, data collection and analysis, decision to publish, or manuscript preparation.

\section{Authors' contributions}

IA collected and analyzed the transcripts and wrote the first draft after the contributions of SG, SH, YH, AY, and HA. SG and HA designed the study and supervised the research coordinators. All authors reviewed the results, the drafts written, and approved the final manuscript submitted.

\section{Acknowledgments}

Not applicable.

\section{References}

1. McGrath J, Saha S, Chant D, Welham J. Schizophrenia: a concise overview of incidence, prevalence, and mortality. Epidemiol Rev. 2008;30:67-76. http://doi.org/10.1093/epirev/mxn001.

2. Judd LL, Akiskal HS, Maser JD, Zeller PJ, Endicott J, Coryell W, Paulus MP, Kunovac JL, Leon AC, Mueller TI, et al. A prospective 12-year study of subsyndromal and syndromal depressive symptoms in unipolar major depressive disorders. Arch Gen Psychiatry. 1998;55(8):694-700. https://www.ncbi.nlm.nih.gov/pubmed/9707379.

3. Siris SG. Suicide and schizophrenia. J Psychopharmacol. 2001;15(2):127-35. http://doi.org/10.1177/026988110101500209.

4. Reine G, Lancon C, Di Tucci S, Sapin C, Auquier P. Depression and subjective quality of life in chronic phase schizophrenic patients. Acta Psychiatr Scand. 2003;108(4):297-303. https://www.ncbi.nlm.nih.gov/pubmed/12956831.

5. Norholm V, Bech P. Quality of life in schizophrenic patients: association with depressive symptoms. Nord J Psychiatry. 2006;60(1):32-7. http://doi.org/10.1080/08039480500504966. 
6. Bressan RA, Chaves AC, Pilowsky LS, Shirakawa I, Mari JJ. Depressive episodes in stable schizophrenia: critical evaluation of the DSM-IV and ICD-10 diagnostic criteria. Psychiatry Res. 2003;117(1):47-56. https://www.ncbi.nlm.nih.gov/pubmed/12581820.

7. Rocca P, Bellino S, Calvarese P, Marchiaro L, Patria L, Rasetti R, Bonetto F. Depressive and negative symptoms in schizophrenia: different effects on clinical features. Compr Psychiatry 2005, 46(4):304-310. https://www.ncbi.nlm.nih.gov/pubmed/16175763.

8. Moller HJ. Occurrence and treatment of depressive comorbidity/cosyndromality in schizophrenic psychoses: conceptual and treatment issues. World J Biol Psychiatry. 2005;6(4):247-63. http://doi.org/10.1080/15622970500316674.

9. Maggini C, Raballo A. Exploring depression in schizophrenia. Eur Psychiatry. 2006;21(4):227-32. http://doi.org/10.1016/j.eurpsy.2005.07.001.

10. Addington D, Addington J, Magicka-Tyndale E. Assessing depression in schizophrenia: the Calgary Depression Scale. Br J Psychiatry Suppl 1993(22):39-44.

https://www.ncbi.nlm.nih.gov/pubmed/8110442.

11. Addington D, Addington J, Magicka-Tyndale E. Specificity of the Calgary Depression Scale for schizophrenics. Schizophr Res. 1994;11(3):239-44.

https://www.ncbi.nlm.nih.gov/pubmed/8193062.

12. Bernard D, Lancon C, Auquier P, Reine G, Addington D. Calgary Depression Scale for Schizophrenia: a study of the validity of a French-language version in a population of schizophrenic patients. Acta Psychiatr Scand. 1998;97(1):36-41. https://www.ncbi.nlm.nih.gov/pubmed/9504701.

13. Muller MJ, Marx-Dannigkeit P, Schlosser R, Wetzel H, Addington D, Benkert $O$. The Calgary Depression Rating Scale for Schizophrenia: development and interrater reliability of a German version (CDSS-G). J Psychiatr Res. 1999;33(5):433-43. https://www.ncbi.nlm.nih.gov/pubmed/10504012.

14. Kaneda Y, Ohmori T, Addington D. [The Japanese version of the Calgary Depression Scale for Schizophrenics (JCDSS)]. No To Shinkei 2000, 52(2):163-166. https://www.ncbi.nlm.nih.gov/pubmed/10723756.

15. Kontaxakis VP, Havaki-Kontaxaki BJ, Margarita MM, Stamoulis SS, Kollias CT, Angelopoulos EK, Christodoulou GN. The Greek version of the Calgary depression scale for schizophrenia. Psychiatry Res. 2000;94(2):163-71. https://www.ncbi.nlm.nih.gov/pubmed/10808041.

16. Schuetze T, Norholm V, Raabaek Olsen L, Hougaard H, Ekstrom M, Wagn P, Bech P. Reliability and validity of the Danish version of the Calgary Depression Scale for Schizophrenia. Nord J Psychiatry. 2001;55(2):119-22. http://doi.org/10.1080/08039480151108543.

17. Hani Y, Ghuloum S, Mahfoud Z, Opler M, Khan A, Yehya A, Abdulhakam A, Hammoudeh S, Al-Mujalli A, Elsherbiny R, et al. Validation of the Arabic Version of Calgary Depression Scale for Schizophrenia. PLoS One. 2016;11(9):e0162304. http://doi.org/10.1371/journal.pone.0162304.

18. Roy A. Depression, attempted suicide, and suicide in patients with chronic schizophrenia. Psychiatr Clin North Am. 1986;9(1):193-206. https://www.ncbi.nlm.nih.gov/pubmed/2421271. 
19. Drake RE, Gates C, Whitaker A, Cotton PG. Suicide among schizophrenics: a review. Compr Psychiatry. 1985;26(1):90-100. https://www.ncbi.nlm.nih.gov/pubmed/3881217.

20. Fenton WS. Depression, suicide, and suicide prevention in schizophrenia. Suicide Life Threat Behav. 2000;30(1):34-49. https://www.ncbi.nlm.nih.gov/pubmed/10782717.

21. Weissman MM, Bland RC, Canino GJ, Greenwald S, Hwu HG, Joyce PR, Karam EG, Lee CK, Lellouch J, Lepine JP, et al. Prevalence of suicide ideation and suicide attempts in nine countries. Psychol Med. 1999;29(1):9-17. https://www.ncbi.nlm.nih.gov/pubmed/10077289.

22. Vijayakumar L. Suicide and mental disorders in Asia. Int Rev Psychiatry. 2005;17(2):109-14. http://doi.org/10.1080/09540260500074735.

23. Lindenmayer JP, Czobor P, Alphs L, Nathan AM, Anand R, Islam Z, Chou JC, InterSe PTSG. The InterSePT scale for suicidal thinking reliability and validity. Schizophr Res. 2003;63(1-2):161-70. https://www.ncbi.nlm.nih.gov/pubmed/12892870.

24. Meltzer HY. Clozapine: is another view valid? Am J Psychiatry. 1995;152(6):821-5. http://doi.org/10.1176/ajp.152.6.821.

25. Hammoudeh S, Ghuloum S, Mahfoud Z, Opler M, Khan A, Yehya A, Abdulhakam A, Al-Mujalli A, Hani $\mathrm{Y}$, Elsherbiny $\mathrm{R}$, et al. Reliability, validity and factorial structure of the Arabic version of the international suicide prevention trial (InterSePT) scale for suicidal thinking in schizophrenia patients in Doha, Qatar. BMC Psychiatry. 2016;16(1):437. http://doi.org/10.1186/s12888-016-1155-4.

26. Siris SG. Diagnosis of secondary depression in schizophrenia: implications for DSM-IV. Schizophr Bull. 1991;17(1):75-98. https://www.ncbi.nlm.nih.gov/pubmed/2047791.

27. Kendell R, Jablensky A. Distinguishing between the validity and utility of psychiatric diagnoses. Am J Psychiatry. 2003;160(1):4-12. http://doi.org/10.1176/appi.ajp.160.1.4.

28. Waring EM, Neufeld RW, Schaefer B. The thought disorder questionnaire. Can J Psychiatry. 2003;48(1):45-51. http://doi.org/10.1177/070674370304800109.

29. Atkinson $\mathrm{M}$, Zibin $\mathrm{S}$, Chuang $\mathrm{H}$. Characterizing quality of life among patients with chronic mental illness: a critical examination of the self-report methodology. Am J Psychiatry. 1997;154(1):99-105. http://doi.org/10.1176/ajp.154.1.99.

30. Favazza AR, Oman M. Overview: foundations of cultural psychiatry. Am J Psychiatry. 1978;135(3):293-303. http://doi.org/10.1176/ajp.135.3.293.

31. Youngstrom E, Weist MD, Albus KE. Exploring violence exposure, stress, protective factors and behavioral problems among inner-city youth. Am J Community Psychol. 2003;32(1-2):115-29. https://www.ncbi.nlm.nih.gov/pubmed/14570441.

32. Estroff SE, Penn DL, Toporek JR. From stigma to discrimination: an analysis of community efforts to reduce the negative consequences of having a psychiatric disorder and label. Schizophr Bull 2004, 30(3):493-509. https://www.ncbi.nlm.nih.gov/pubmed/15631242.

33. Al-Krenawi A, Graham JR, Kandah J. Gendered utilization differences of mental health services in Jordan. Community Ment Health J 2000, 36(5):501-511. https://www.ncbi.nlm.nih.gov/pubmed/10994683. 
34. Fakhr El-Islam M. Arab culture and mental health care. Transcult Psychiatry. 2008;45(4):671-82. http://doi.org/10.1177/1363461508100788.

35. Palinkas LA. Qualitative and mixed methods in mental health services and implementation research. J Clin Child Adolesc Psychol. 2014;43(6):851-61. http://doi.org/10.1080/15374416.2014.910791.

36. Room R, Janca A, Bennett LA, Schmidt L, Sartorius N. WHO cross-cultural applicability research on diagnosis and assessment of substance use disorders: an overview of methods and selected results. Addiction. 1996;91(2):199-220. https://www.ncbi.nlm.nih.gov/pubmed/8835277. discussion 221 130.

37. Qatar Statistics. Authority. Census 2016, [www.qsa.gov.qa.].

38. Braun V, Clarke V. Using thematic analysis in psychology. Qualitative Research in Psychology. 2006;3(2):77-101. http://doi.org/10.1191/1478088706qp063oa.

39. Bhui K, Mohamud S, Warfa N, Craig TJ, Stansfeld SA. Cultural adaptation of mental health measures: improving the quality of clinical practice and research. Br J Psychiatry. 2003;183:184-6. http://doi.org/10.1192/bjp.183.3.184.

40. Jablensky A, Sartorius N, Ernberg G, Anker M, Korten A, Cooper JE, Day R, Bertelsen A. Schizophrenia: manifestations, incidence and course in different cultures. A World Health Organization ten-country study. Psychol Med Monogr Suppl. 1992;20:1-97. http://doi.org/10.1017/s0264180100000904.

41. Sartorius N, Jablensky A, Korten A, Ernberg G, Anker M, Cooper JE, Day R. Early manifestations and first-contact incidence of schizophrenia in different cultures. A preliminary report on the initial evaluation phase of the WHO Collaborative Study on determinants of outcome of severe mental disorders. Psychol Med. 1986;16(4):909-28. http://doi.org/10.1017/s0033291700011910.

42. Malterud K. Qualitative research: standards, challenges, and guidelines. Lancet. 2001;358(9280):483-8. http://doi.org/10.1016/S0140-6736(01)05627-6.

43. Eskin M, AlBuhairan F, Rezaeian M, Abdel-Khalek AM, Harlak H, El-Nayal M, Asad N, Khan A, Mechri A, Noor IM, et al: Suicidal Thoughts, Attempts and Motives Among University Students in 12 MuslimMajority Countries. Psychiatr Q 2019, 90(1):229-248. http://doi.org/10.1007/s11126-018-9613-4.

44. Vignoles VL, Owe E, Becker M, Smith PB, Easterbrook MJ, Brown R, Gonzalez R, Didier N, Carrasco D, Cadena MP, et al. Beyond the 'east-west' dichotomy: Global variation in cultural models of selfhood. J Exp Psychol Gen. 2016;145(8):966-1000. http://doi.org/10.1037/xge0000175.

45. Zolezzi M, Alamri M, Shaar S, Rainkie D. Stigma associated with mental illness and its treatment in the Arab culture: A systematic review. Int J Soc Psychiatry 2018, 64(6):597-609. http://doi.org/10.1177/0020764018789200.

46. Rassool GH. Cultural Competence in Counseling the Muslim Patient: Implications for Mental Health. Arch Psychiatr Nurs. 2015;29(5):321-5. http://doi.org/10.1016/j.apnu.2015.05.009.

47. Sabry WM, Vohra A. Role of Islam in the management of Psychiatric disorders. Indian J Psychiatry. 2013;55(Suppl 2):205-14. http://doi.org/10.4103/0019-5545.105534.

48. Lee KK, Yamada AM, Kim MA, Dinh TQ. Interdependent recovery of adults with schizophrenia: Asian American consumer perspectives of family involvement and influence. Psychiatr Rehabil J 2015, 
38(3):273-275. http://doi.org/10.1037/prj0000076.

49. Villares CC, Redko CP, Mari JJ. Conceptualizations of illness among relatives of patients diagnosed with schizophrenia. Int Rev Psychiatry. 2017;29(3):230-40.

http://doi.org/10.1080/09540261.2017.1285549.

50. Bertolote JM, Fleischmann A, De Leo D, Bokhari J, Botega N, De Silva D, Tran Thi Thanh H, Phillips M, Schlebusch L, Varnik A, et al. Suicide attempts, plans, and ideation in culturally diverse sites: the WHO SUPRE-MISS community survey. Psychol Med. 2005;35(10):1457-65. http://doi.org/10.1017/S0033291705005404.

51. Shah A, Chandia M. The relationship between suicide and Islam: a cross-national study. J Inj Violence Res. 2010;2(2):93-7. http://doi.org/10.5249/jivr.v2i2.60.

52. Xiang YT, Weng YZ, Leung CM, Tang WK, Ungvari GS. Sociodemographic and clinical correlates of lifetime suicide attempts and their impact on quality of life in Chinese schizophrenia patients. $J$ Psychiatr Res. 2008;42(6):495-502. http://doi.org/10.1016/j.jpsychires.2007.06.001.

53. Bhatia T, Thomas P, Semwal P, Thelma BK, Nimgaonkar VL, Deshpande SN. Differing correlates for suicide attempts among patients with schizophrenia or schizoaffective disorder in India and USA. Schizophr Res. 2006;86(1-3):208-14. http://doi.org/10.1016/j.schres.2006.04.015.

54. Bassiouney R. Arabic Sociolinguistics. In. Edinburgh: Edinburgh University Press; 2009. 1 online resource (337 pages).

55. Nydell M. Understanding Arabs. Yarmouth: Intercultural Press; 1987.

56. Barnes A. Race, schizophrenia, and admission to state psychiatric hospitals. Adm Policy Ment Health. 2004;31(3):241-52. http://doi.org/10.1023/b:apih.0000018832.73673.54.

57. Alarcon RD, Westermeyer J, Foulks EF, Ruiz P. Clinical relevance of contemporary cultural psychiatry. J Nerv Ment Dis. 1999;187(8):465-71. https://www.ncbi.nlm.nih.gov/pubmed/10463063.

\section{Supplementary Files}

This is a list of supplementary files associated with this preprint. Click to download.

- AppendixIBMCsubthematicanalysis.docx 